\title{
Experimental Analysis \\ of a Directive Antenna with a 3D-EBG Superstrate
}

\author{
Lara Pajewski $^{1}$, Fabrizio Frezza ${ }^{1}$, Marian Marciniak ${ }^{2}$, Emanuele Piuzzi ${ }^{1}$, and Giorgia V. Rossi ${ }^{3}$ \\ ${ }^{1}$ Department of Information Engineering, Electronics and Telecommunications, Sapienza University of Rome, Rome, Italy \\ ${ }^{2}$ National Institute of Telecommunications, Warsaw, Poland \\ ${ }^{3}$ Imperial College London, London, United Kingdom
}

https://doi.org/10.26636/jtit.2017.120017

\begin{abstract}
A three-dimensional electromagnetic crystal is employed as a directivity-enhancing superstrate for planar antennas. The crystal is a woodpile made of alumina rods. In a shielded anechoic chamber, the performance of a patch antenna covered with the woodpile is measured. The superstrate is positioned at different distances from the antenna and its orientation is varied in the $8-12 \mathbf{~ G H z}$ frequency range. The return loss, gain and radiation pattern in the $\mathrm{E}$ - and $\mathrm{H}$-planes are measured. The electromagnetic behavior of Fabry-Perot cavities with woodpile mirrors, equivalent to the compound radiator, is also studied. The main effect of the crystal on the antenna performance is an enhancement of about $10 \mathrm{~dB}$ in maximum gain. A rather complete series of experiments is presented, highlighting the role of the periodic structure in the directivity enhancement and allowing a deeper understanding of the electromagnetic phenomena involved in EBG resonator antennas. Benefits and disadvantages of this kind of antennas are discussed and ideas for future research are given.
\end{abstract}

Keywords-directivity enhancement, electromagnetic bandgap materials, Fabry-Perot cavities, periodic structures, woodpile.

\section{Introduction}

Electromagnetic band gap (EBG) materials [1] can be successfully employed to improve the performance of antennas [2], [3]. When used as planar reflectors [4], substrates [5], or high-impedance ground-planes [6], are able to eliminate the drawbacks of conducting ground-planes, to prevent the propagation of surface waves also allowing a lowering of the antenna profile, and to improve the radiation efficiency. In EBG resonator antennas [7]-[11], an electromagnetic crystal is employed as a superstrate on a primary radiator, backed with a ground plane, and its main effect is a considerable increase in the directivity. Else, it is possible to obtain a highly-directive antenna by embedding a source in an EBG working near its band gap [12], thanks to the limited angular propagation allowed within the crystal. Recently, these two different methods for antennadirectivity enhancement were compared, with reference to several optimized two-dimensional configurations based on either square or triangular lattices of dielectric rods [13].
Several research teams have been studying the EBG resonator antenna as a possible solution to be adopted when a directive beam is needed. This is an interesting alternative to aperture antennas and antenna arrays, free of some problems inherent to the usual directive systems, such as the size of the focal structures and the limitations/complications induced by the feeding circuit of arrays.

In EBG resonator antennas, the periodic cover is placed at a distance equal to an integer multiple of half a wavelength from the source. Forward radiation can be remarkably enhanced by means of in-phase multiple reflections. A single feed is usually employed, allowing the gain to be increased with low complexity. In some cases [14], arrays of patches or horns were used as sources. Systems operating at different frequencies, ranging from the microwave region to millimeter waves, can be designed and realized. Electromagnetic crystals with one, two or three periodicity directions can be employed as superstrates.

In [15], the characteristics of directivity enhancement using EBGs, frequency-selective surfaces and left-handed metamaterials were compared. A Fabry-Perot cavity can be used as a cover, in spite of a single EBG superstrate [14]. Usually, the cover is a planar structure. In [16], a dipole source embedded in a cylindrical EBG was studied, by using a rigorous semi-analytical method, and the performances of the compound antenna were numerically investigated for three different EBG configurations as well as varying the dipole position inside the periodic structure.

In this paper, we use a woodpile cover to increase the gain of a microstrip-patch antenna. The woodpile is a threedimensional (3D) EBG with a rather simple geometry, that may present complete 3D stop-bands [17], [18]. It consists of a stack of dielectric rods with alternating orthogonal orientations. The cross-section of the rods may be rectangular or circular, or else air cylindrical holes may be drilled in a dielectric sample. A woodpile with square-section rods is often preferred, because easier to fabricate.

The aim of our paper is to complement the work published in [11], by presenting several new experimental results. These results highlight the role of the periodic superstrate in the directivity enhancement and we believe that they al- 
low researchers interested in EBG resonator antennas to achieve a deeper understanding of the electromagnetic phenomena involved in this kind of structures. It is worth noting that experimental works at microwave frequencies, regarding the use of EBGs in antenna engineering, are not so frequent. In most of the published papers numerical results of simulations are reported.

In Section 2, proposed EBG resonator antenna is described. We designed a woodpile, with a complete band gap centered on $f=12 \mathrm{GHz}$ and extending over almost $4 \mathrm{GHz}$ [19]. The design was carried out by using an in-house code that implements the Fourier Modal Method (FMM) [20]. Woodpile samples were fabricated, by means of alumina rods with square cross-section [11]. A rectangular patch antenna was realized, resonating at $10.3 \mathrm{GHz}$. A supporting structure was specifically designed and realized, to hold the antenna together with its woodpile cover during experimental investigations.

Section 3 is devoted to numerical results. In a shielded anechoic chamber, in the $8-12 \mathrm{GHz}$ frequency range, the return loss, gain, and radiation pattern were measured in the E- and H-planes, of the compound antenna. The superstrate was positioned at different distances from the patch and its orientation was varied. The electromagnetic behavior of woodpile Fabry-Perot cavities, equivalent to the EBG resonator antenna, was also measured.

Conclusions are drawn in Section 4, where benefits and disadvantages of the EBG resonator antenna are also discussed, new applications of these radiators are suggested and ideas for future research are given.

\section{The EBG Resonator Antenna}

A woodpile crystal was synthesized, by using an in-house code implementing the FMM, a full-wave approach that solves the monochromatic plane-wave scattering problem by dielectric finite-thickness crossed gratings, as proposed in [20]. The design is described in [19] and resumed in [11], where the electromagnetic behavior of the proposed woodpile is also analyzed.

The crystal consists of four periodic layers of alumina rods, with square section having side length $w=3.18 \mathrm{~mm}$. The relative dielectric constant of the rods is $\varepsilon_{\mathrm{r}, \mathrm{al}}=9.8$. The spacing of each layer is $d=8 \mathrm{~mm}$. Rods belonging to consecutive layers are orthogonal. In the third and fourth layers, the rods have the same orientation as in the first and second ones, respectively, but they are in offset by half of the horizontal spacing (see Fig. 1). We used the above-mentioned FMM code to simulate a monochromatic plane-wave with electric/magnetic field parallel to the rods impinging normally on this crystal. Results show that there is a band gap centered on $f=12 \mathrm{GHz}$, extending over almost $4 \mathrm{GHz}$. By studying the off-plane behavior of the woodpile, we found that the band gap is scarcely sensitive to variations of the electromagnetic-field incidence and polarization. Only a slight reduction in depth and width of the band gap occurs, when these parameters are changed.
Two identical woodpile samples were realized. The rod length is $20 \mathrm{~cm}$, that is almost seven wavelengths at $10 \mathrm{GHz}$, and is equal to $25 \mathrm{~d}$. This ensures that the sample behavior is close to that of an electromagnetic crystal with rods of infinite length and with an infinite number of periods in both the directions of the rod axes. The diameter tolerance of the rods is $\pm 3 \%$, their straightness is represented by a camber/length parameter $\leq 0.003$, and the maximum twist is $2^{\circ}$ per $30.48 \mathrm{~cm}$. For more details about the woodpile fabrication see [11].

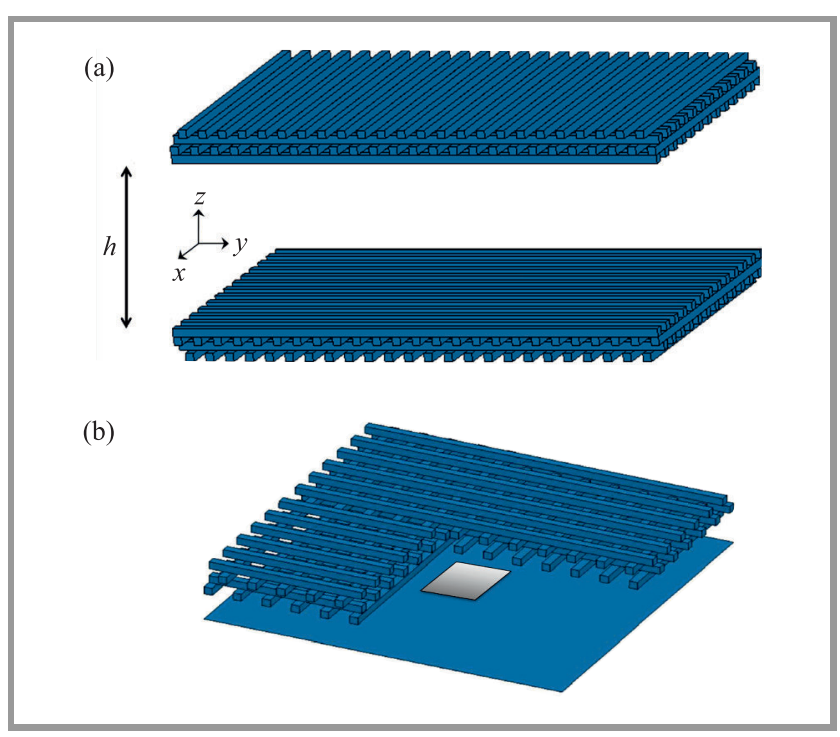

Fig. 1. Woodpile cavity (a), woodpile-covered antenna (b).

We realized a microstrip antenna, from a $0.76 \mathrm{~mm}$ thick Rogers/RT Duroid 5870 laminate (relative permittivity 2.33 ), printed on both sides with $36-\mu \mathrm{m}$ thick copper. With a PC-controlled milling table, on one side of the layer a $8 \times 8.4 \mathrm{~mm}$ rectangular patch was cut. The antenna is fed from below by a coaxial probe (SMA connector). The feed point, where the probe is attached to the patch, is centered with respect to the shorter side of the patch, and it is $1.2 \mathrm{~mm}$ away from the centre of the longer side.

A PVC table and a teflon-PVC support were designed and realized, to hold the couple of woodpile samples, or the antenna and a woodpile, during experimental investigations.

\section{Experimental Results}

All the experiments were performed in a $3.20 \times 3.20 \times$ $2.70 \mathrm{~m}$ shielded anechoic chamber, by using a HP 8530 vector network analyzer. We analyze the electromagnetic behavior of Fabry-Perot cavities with woodpile mirrors, changing the cavity length and the field polarization (Subsection 3.1). We subsequently study the performances of the woodpile-covered patch, changing the distance between the antenna and the EBG cover, and the field polarization (Subsections 3.2-3.4). As many results are reported, it is possible to appreciate the consistence of the experimental 

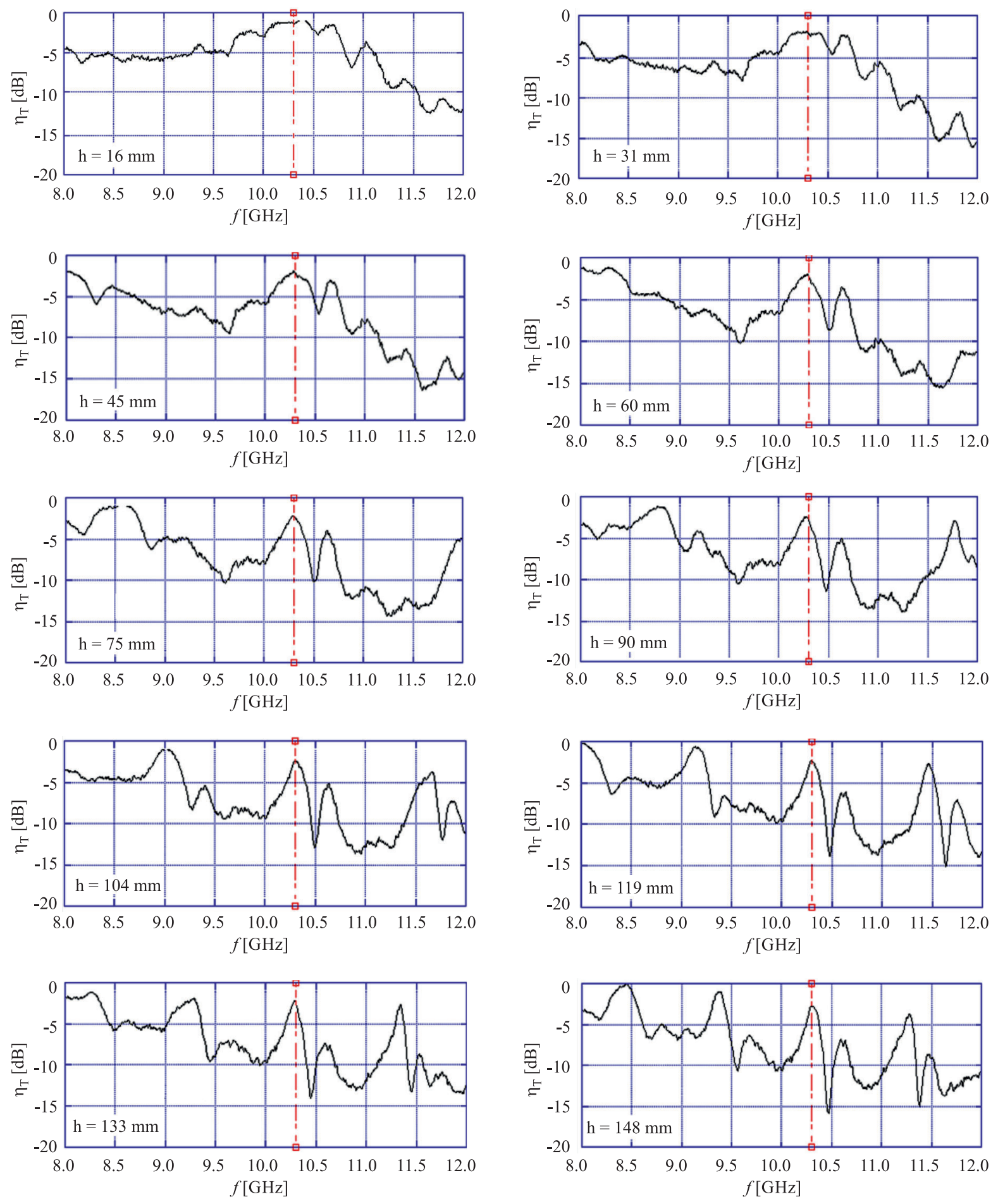

Fig. 2. Transmission efficiency of the cavity, $\eta_{T}$, as a function of frequency, for ten different cavities resonating at $10.3 \mathrm{GHz}$; the electric field is parallel to the internal rods of the woodpile cavity.

data we collected. Moreover, one may achieve a general understanding of the electromagnetic behavior of the considered devices. The effects of the cavity length and of the field polarization on the overall performance of the EBG resonator antenna are clearly pointed our. A sketch of both the Fabry-Perot cavity and the woodpile-patch is reported in Fig. 1.
As a preliminary experiment, we measured the reflection parameter, the gain, and the radiation diagram in the Eand H-planes, of the patch antenna introduced in Section 2. This antenna resonates at $10.3 \mathrm{GHz}$. At this frequency, the magnitude of its return loss is $\left|S_{11}\right|=-11.69 \mathrm{~dB}$. By using, as a standard transmitter, a horn antenna with a maximum gain equal to $16 \mathrm{~dB}$ and a standing-wave ratio (SWR) equal 

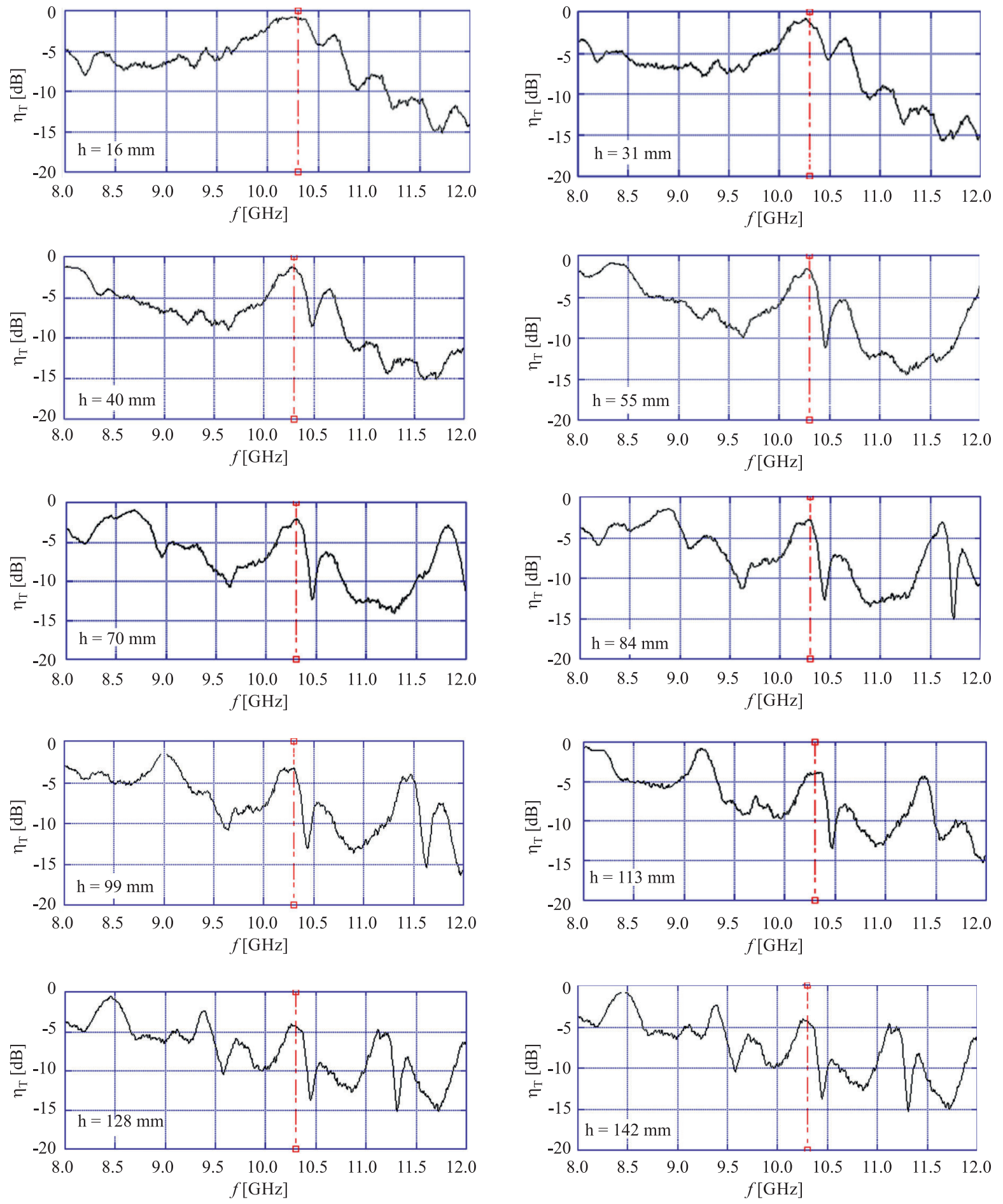

Fig. 3. Transmission efficiency of the cavity, $\eta_{T}$, as a function of frequency, for ten different cavities resonating at $10.3 \mathrm{GHz}$; the electric field is orthogonal to the internal rods of the woodpile cavity.

to 1.25 , we found that the maximum gain of our patch antenna is $G_{\mathrm{p}}=6.18 \mathrm{~dB}$.

\subsection{Fabry-Perot Cavity with Woodpile Mirrors}

We performed a series of measurements on a Fabry-Perot cavity with woodpile mirrors. The adopted setup consisted of a couple of X-band precision pyramidal horn antennas, placed one in front of the other, with both the woodpile samples in the middle. The samples were placed at a distance $h$ from each other and held by the PVC/Teflon support.

This structure resonates when its equivalent length $L_{\mathrm{eq}}$ is an integer multiple of $\frac{\lambda}{2}$, where $\lambda$ is the wavelength of the 
electromagnetic field in air. The cavity may also be considered as an EBG with a break of its periodicity, in which some periodic layers are replaced by a homogeneous airregion with thickness $h$ : the main effect of creating a defect in a band-gap structure, is the occurrence of transmission peaks inside the prohibited bands [21], [22].

We identified a set of cavities with different lengths and showing a transmission peak at $10.3 \mathrm{GHz}$ (resonance frequency of proposed patch antenna). We considered different polarization states of the electromagnetic field (electric field parallel or orthogonal to the most internal rods of the cavity).

In order to comply with the frequency band of the horn antennas, measurements were performed in the $8-12 \mathrm{GHz}$ range. The distance between the horns was chosen after a series of preliminary experiments aimed at ensuring a good compromise between the planarity of the wavefront impinging on the woodpile and the diffraction effects due to the finite extension of the samples.

The cavity transmission properties were determined from the measured $S_{21}$ scattering parameter. Its magnitude is equal to the transmission efficiency of the cavity.

A preliminary reference calibration was performed with no sample placed between the two horns. Upon completion of each experimental session a further measurement was performed removing again the woodpile samples, to check that the drift in the measurement system was within acceptable levels.

In Fig. 2, the measured transmission efficiency $\eta_{T}$ is plotted as a function of frequency, for ten different cavities resonating at $10.3 \mathrm{GHz}$. In all those measurements, the electric field was parallel to the rods of the woodpile layers nearest to the air region inside the cavity. It is noted that in longer cavities a higher number of transmission peaks occurs within the considered frequency range, as expected. Accordingly, the peak centered in $10.3 \mathrm{GHz}$ has a higher quality factor when the cavity is longer. It is also noted that, in all the studied cases (i.e. for all values of the cavity length), the transmission peaks occurring at lower frequencies show a higher efficiency. This is an expected phenomenon and is due to the fact that, when the wavelength of the electromagnetic field is longer, the thickness of the woodpile mirrors is electrically smaller, hence the propagation through the mirrors causes smaller losses.

In Fig. 3, the same as in Fig. 2 is shown, for ten different cavities with electric field orthogonal to the most internal rods of the cavity. Similar comments apply. It can be appreciated that the equivalent length of a woodpile cavity is highly dependent on the electromagnetic field polarization with respect to the EBG orientation. In this regard, please also see [11], where we presented simulation results showing that the periodic arrangement of bars perpendicular to the electric field has a negligible effect on the transmission efficiency through the whole structure.

\subsection{Return Loss of the Woodpile-covered Patch Antenna}

According to the image theory, a configuration equivalent to the Fabry-Perot woodpile cavity is obtained, if the cavity is halved, with respect to its symmetry plane, by means of a perfectly conducting surface. If the ground plane of a patch antenna is employed instead of a perfectly conducting surface, an EBG resonator antenna is built (see Fig. 1b).

The measurements we performed on the woodpile-covered patch antenna include return loss, gain, and radiation patterns in the E- and H- planes. In this Subsection, return-loss results are reported.

For return-loss measurements, an Agilent E8363B performance network analyzer was used, which was preliminarily calibrated at the port by means of a short-open-load procedure, employing the $85052 \mathrm{~B}$ mechanical calibration kit.

The superstrate was positioned at various distances $\frac{h}{2}$ from the patch, corresponding to half length of the cavities resonating at $10.3 \mathrm{GHz}$. We verified that achieving a good parallelism between the ground-plane and the woodpile is very important to maximize the performances of the compound antenna. Moreover, we observed that a woodpile shift, parallel to the ground plane, does not affect the behavior of the radiator. The orientation of the superstrate, instead, is very important, as in woodpile cavities.

In Figs. 4 and 5, the return loss of the woodpile-covered patch antenna is presented. In particular, in Fig. 4 ten different values of $\frac{h}{2}$ are considered and the woodpile rods nearest to the ground plane are parallel to the electric field radiated by the patch. Figure 5 is analogous to Fig. 4, but now the rods nearest to the ground plane are orthogonal to the electric field. For the return loss of the patch alone, see Fig. 15 in [11].

In all the examined cases, we observed that the EBG superstrate does not significantly affect the antenna matching. However, we found that when the equivalent distance between the woodpile and the patch is roughly equal to an integer multiple of $\frac{\lambda}{2}$ (as in the right-column plots of Figs. 4 and 5), the effect of the EBG over the $\left|S_{11}\right|$ frequency trend is stronger than it is when the above-mentioned equivalent distance is an odd integer multiple of $\frac{\lambda}{4}$ (as in the leftcolumn plots of Figs. 4 and 5). This happens because in the former case (right-column plots) the multiple reflections between the antenna and the superstrate are summed in phase. Hence their overall effect on the return loss is more pronounced, whereas in the latter case (left-column plots) the multiple reflections are in phase opposition to each other and their overall effect on the return loss is totally negligible.

\subsection{Gain of the Woodpile-covered Patch Antenna}

Gain and radiation pattern measurements were performed with a setup similar to the one described for cavity characterization. In particular, the receiving horn antenna was replaced by the patch antenna, which was positioned on a remotely controlled turntable. The distance between the two antennas was chosen to be $1 \mathrm{~m}$, in order to be in the 

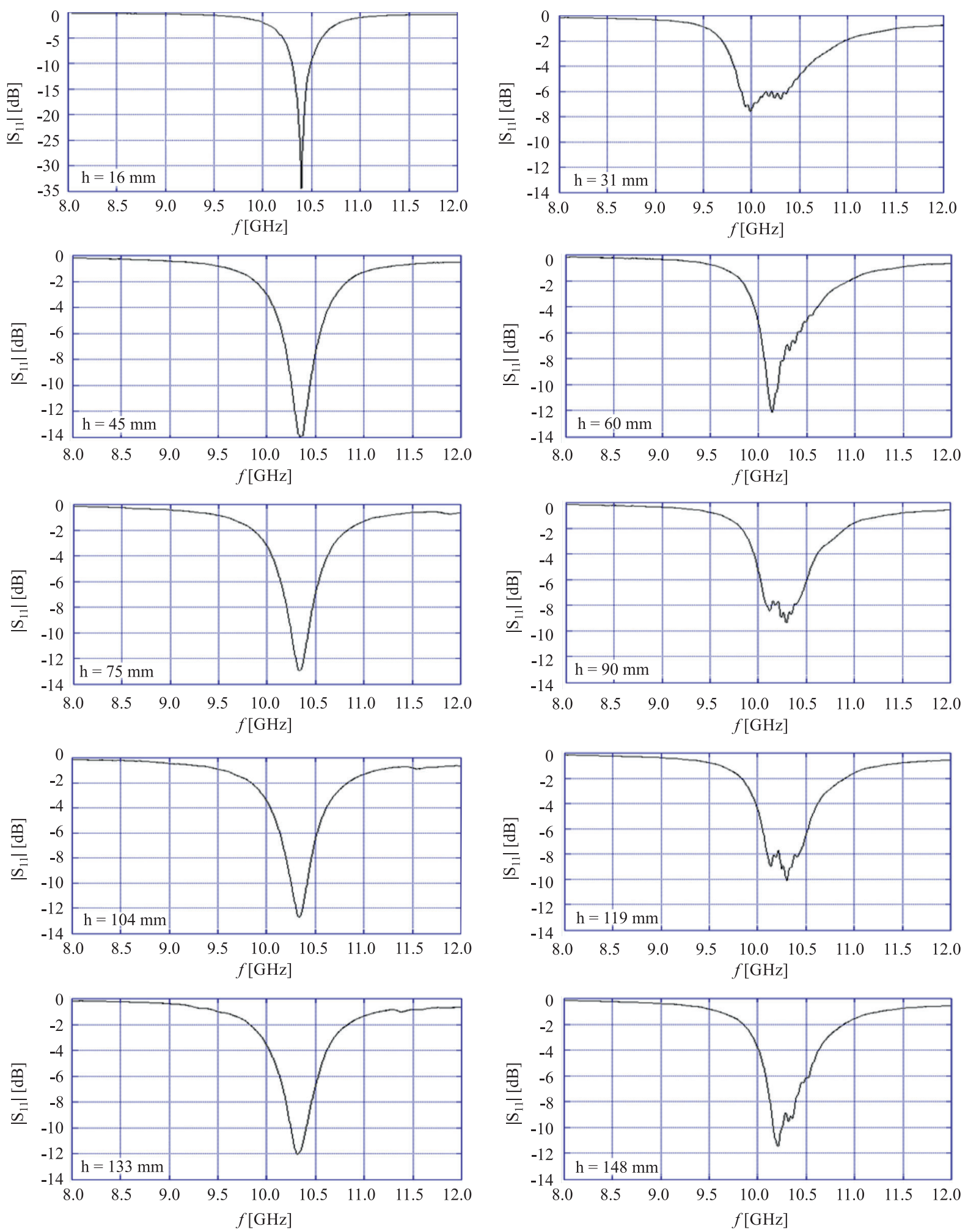

Fig. 4. Return loss of the woodpile-covered patch antenna, vs. frequency. Ten different values of the distance $\frac{h}{2}$ between patch and woodpile are considered. The woodpile rods nearest to the ground plane are parallel to the electric field radiated by the patch.

far field of the radiators. The setup was preliminarily subjected to a reference calibration with the microstrip antenna replaced by the second standard gain horn. In this Subsection, gain results are presented.
In Fig. 6, the maximum gain of the woodpile-covered patch antenna, $G_{\mathrm{pw}}$, normalized to the maximum gain of the patch alone, $G_{\mathrm{p}}$, is plotted as a function of frequency, for the same woodpile orientation and $\frac{h}{2}$ distances studied 

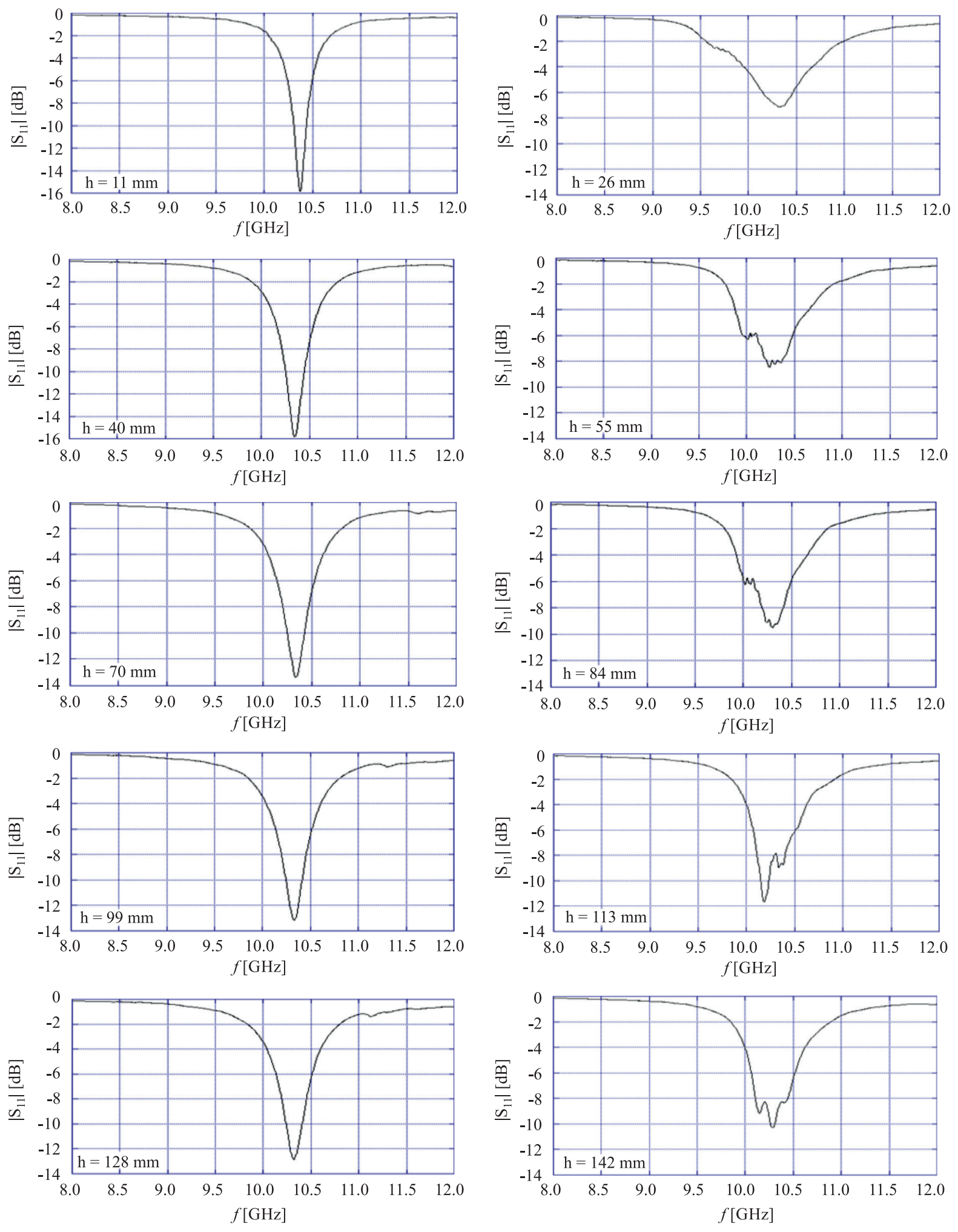

Fig. 5. Return loss of the woodpile-covered patch antenna, vs. frequency. Ten different values of the distance $\frac{h}{2}$ between patch and woodpile are considered. The woodpile rods nearest to the ground plane are orthogonal to the electric field radiated by the patch.

in Fig. 4. When the equivalent distance between the woodpile and the patch is roughly equal to an integer multiple of $\frac{\lambda}{2}$, as in the right column of the figure, the maximum gain enhancement due to the woodpile occurs at $10.3 \mathrm{GHz}$.
The multiple reflections are present between the antenna and the superstrate, and they are summed in phase at the interface between air and woodpile. Instead, when the abovementioned equivalent distance is an odd integer multiple 

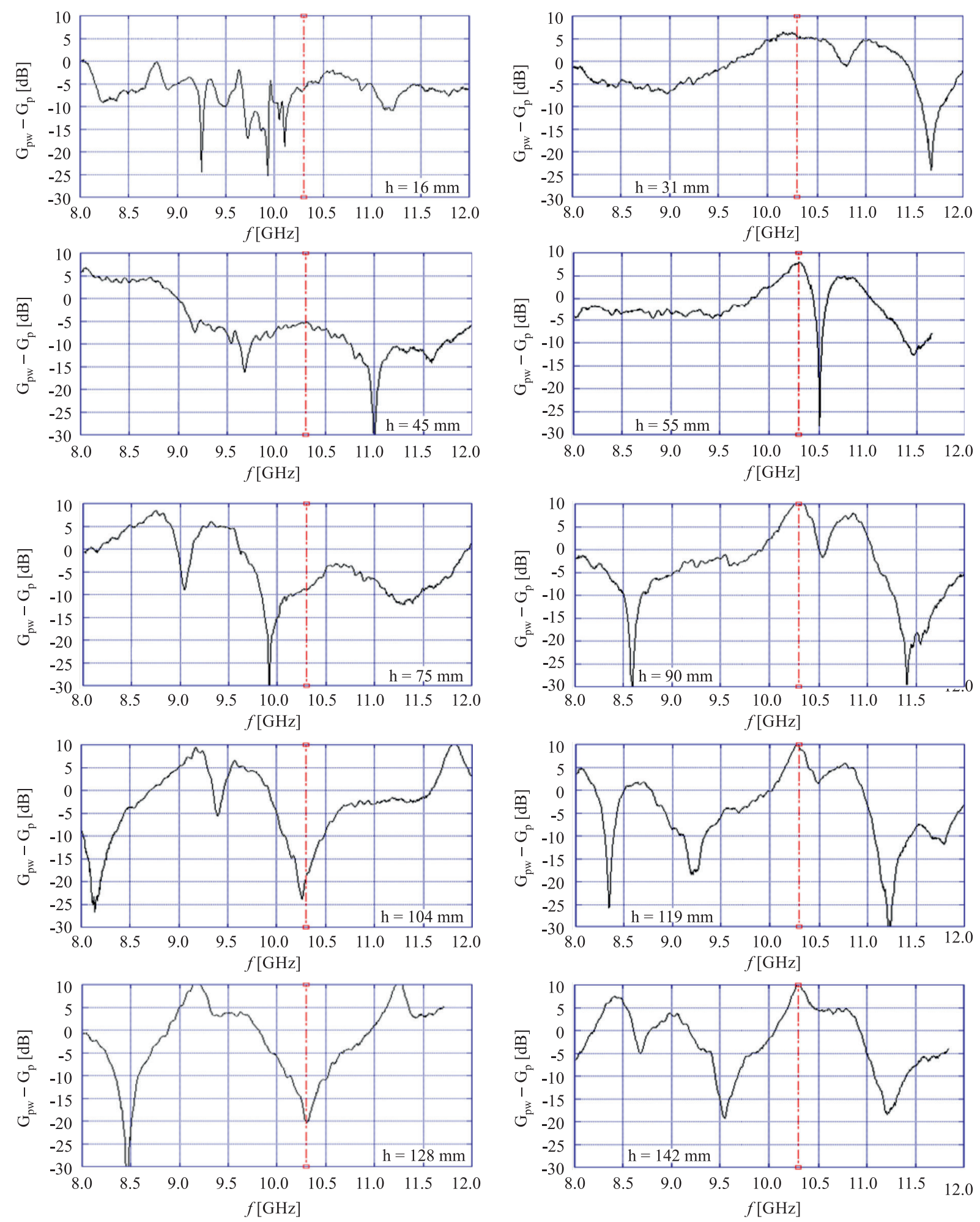

Fig. 6. Maximum gain of the woodpile-covered patch antenna, $G_{\mathrm{pw}}$, normalized to the maximum gain of the patch alone, $G_{\mathrm{p}}$, in decibels, vs. frequency; the distance $\frac{h}{2}$ assumes ten different values, the woodpile bars nearest to the ground plane are parallel to the electric field radiated by the patch.

of $\frac{\lambda}{4}$, as in the left column of the figure, at $10.3 \mathrm{GHz}$ the effect of the woodpile is a strong reduction of the gain. The multiple reflections arrive at the woodpile in phase opposition to each other.
Similar results were collected for the configurations with the bars nearest to the ground plane parallel or orthogonal to the electric field, as can be appreciated by comparing Fig. 6 with Fig. 7. 

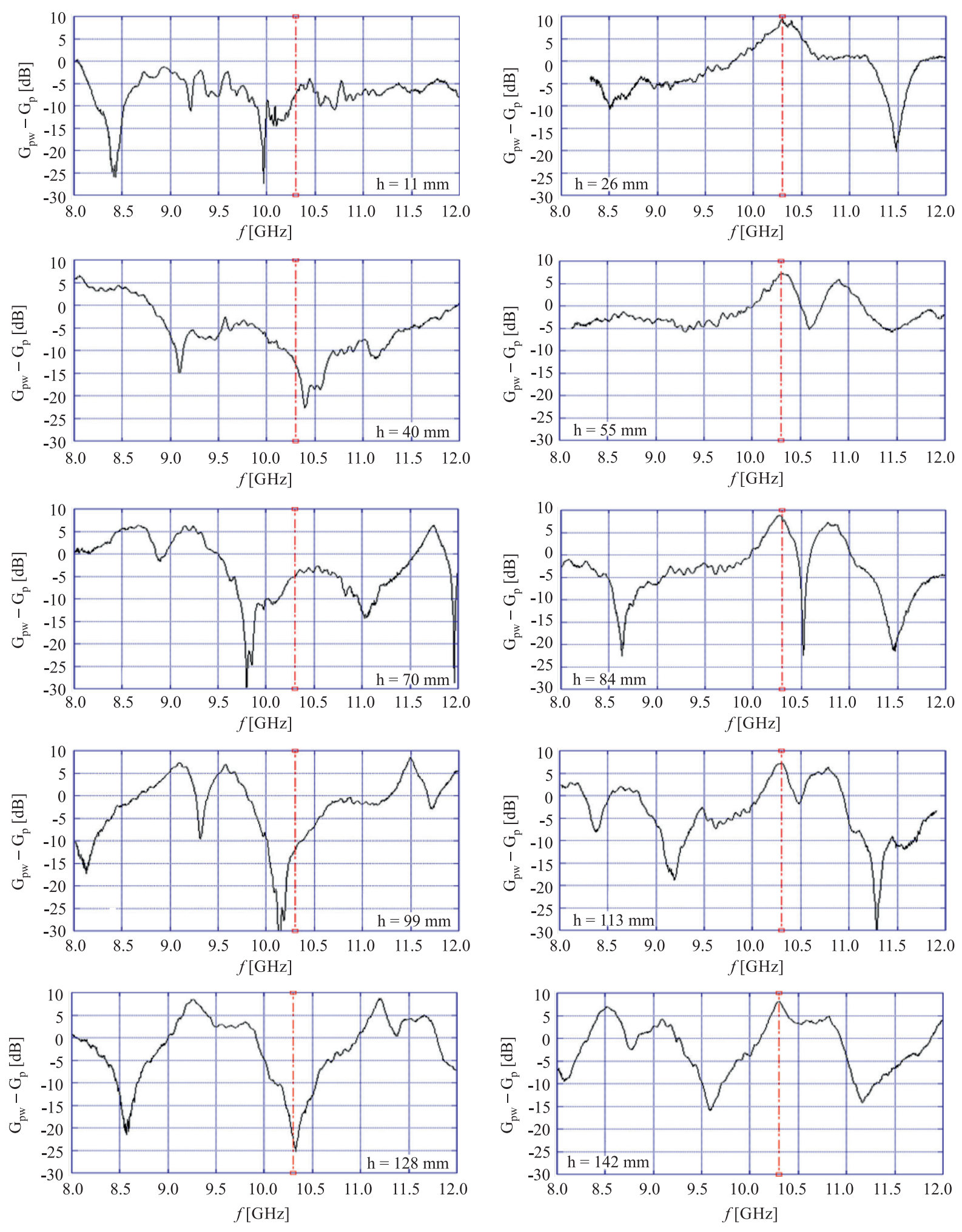

Fig. 7. Maximum gain of the woodpile-covered patch antenna, $G_{\mathrm{pw}}$, normalized to the maximum gain of the patch alone, $G_{\mathrm{p}}$, in decibels, vs. frequency; the distance $\frac{h}{2}$ assumes ten different values, the woodpile bars nearest to the ground plane are orthogonal to the electric field radiated by the patch.

The gain enhancement at $10.3 \mathrm{GHz}$ turns out to be equal to about $10 \mathrm{~dB}$, regardless of the distance between patch and woodpile. Note that, in some cases, we measured a smaller gain enhancement. This is due to the fact that achieving a good parallelism between the ground plane and the woodpile is extremely important for maximizing the per- 


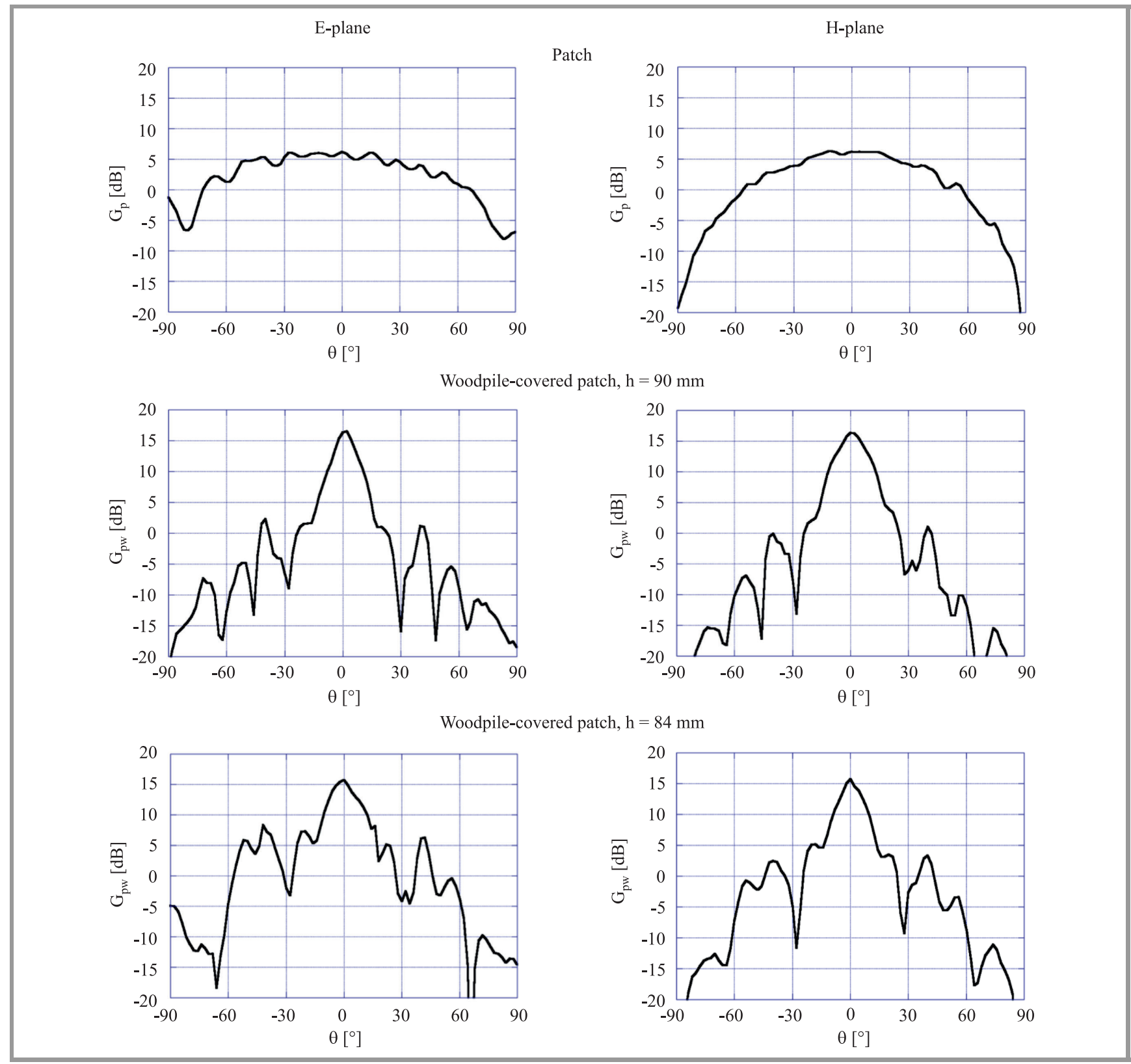

Fig. 8. E- and H-plane radiation pattern for the patch alone, for the woodpile-covered patch with $h=90 \mathrm{~mm}$ (configuration studied in Fig. 6), and for the woodpile-covered patch with $h=84 \mathrm{~mm}$ (configuration studied in Fig. 7), at $10.3 \mathrm{GHz}$.

formance of the compound antenna, but unfortunately we do not have the necessary experimental means for checking and optimizing the parallelism.

\subsection{Radiation Pattern of the Woodpile-covered Patch Antenna}

We finally measured the E- and H-plane radiation patterns of the woodpile-covered patch antenna at $10.3 \mathrm{GHz}$. In Fig. 8, a couple of examples are reported. In particular, we present results for the patch alone, for the woodpilecovered patch with $h=90 \mathrm{~mm}$ (configuration studied in Fig. 6), and for the woodpile-covered patch with $h=84 \mathrm{~mm}$ (configuration studied in Fig. 7). Data were recorded every $2^{\circ}$. The directivity enhancement due to the presence of the EBG cover can be easily appreciated.

\section{Conclusions}

This work dealt with EBG resonator antennas. A woodpile was used for directivity-enhancing of a linearly-polarized rectangular-patch antenna. The compound radiating system was experimentally characterized in the $8-12 \mathrm{GHz}$ frequency range, in a shielded anechoic chamber, by using a vector network analyzer.

The EBG superstrate was designed by using an in-house code implementing the Fourier Modal Method. Two wood- 
pile samples were fabricated, by means of alumina rods with square cross-section. Moreover, a rectangular patch antenna was realized.

First, the transmission properties of Fabry-Perot resonators with woodpile mirrors were measured. The cavity length and the orientation of the mirrors were varied. In particular, we looked for configurations resonating at $10.3 \mathrm{GHz}$, i.e. at the resonance frequency of the patch antenna.

Subsequently, the return loss, the gain, and the radiation pattern in the E- and H-planes were measured, for the patch antenna covered with the woodpile. The superstrate was positioned at different distances from the antenna and its orientation was varied. In order to obtain a directivity enhancement the equivalent distance between the woodpile and the antenna had to be an integer multiple of $\frac{\lambda}{2}$. With such setup, the presence of the EBG superstrate above the patch determined an enlargement of the source equivalentaperture area, resulting in highly directive radiation. The gain of the woodpile-covered patch turned out to be about $10 \mathrm{~dB}$ higher than the gain of the patch alone. Similar results were obtained for configurations with the bars nearest to the patch parallel or orthogonal to the electric field. In all the examined cased, the periodic superstrate did not significantly affect the antenna matching.

An interesting feature of this kind of radiating systems is that they are more compact than classical highly-directive antennas. Moreover, they can be excited by a single feeding device. Note that achieving a high directivity by means of an array of sources is more expensive. Also that typically an array has a highly resonant matching.

From our results it is evident that, once the distance between ground-plane and woodpile is fixed, there are different resonance frequencies at which the gain enhancement occurs. This suggests that a number of primary radiators, working at appropriate frequencies, may benefit from the same superstrate to increase their directivity.

We also observed that the resonance frequency of the compound antenna strongly depends on the source polarization, and rods orthogonal to the electric-field direction have a very little influence on the antenna performances. Hence, orthogonal radiators might exploit different layers of the same EBG cover. This means that it is possible to design a device generating or receiving two orthogonal uncoupled linear polarization states, with high directivity, or else, a device allowing an easy control of the polarization of the emitted or received field.

Further ideas for future work include: the design and realisation of EBG resonator antennas with low-thickness superstrates, in order to reduce the radiating-system size or, with monolithic superstrates, easier to implement with respect to a woodpile and less fragile; else, with low-permittivity and low-cost superstrates, to be fabricated with common $3 \mathrm{D}$ printers. With respect to the latter, we plan to investigate the possibility to realise heterogeneous superstrates, in which different permittivity values can be achieved from a single material. Our idea is to create small (compared to the wavelength) air inclusions in the material, so that - by varying the size and periodicity of the small voids - the local permittivity of the superstrate can be controlled.

The authors are keen to develop these innovative applications in the near future.

\section{Acknowledgements}

This study was carried out in the framework of the PRIN (National Interest Research Programs) Project "Development of design procedures and strategies for frequency selective structures," funded by the Italian Government.

This work strongly benefited from the networking activities carried out in the COST (European Cooperation in Science and Technology) Actions MP0702 "Towards Functional Sub-Wavelength Photonic Structures" (cost-mp0702.nit.eu) and TU1208 "Civil engineering applications of Ground Penetrating Radar" (www.GPRadar.eu). The authors are deeply grateful to COST (www.cost.eu) for funding and supporting the Actions MP0702 and TU1208. This paper is a contribution to the JTIT Special Issue "Recent Progress in Electromagnetic Theory and its Applications" organized by the COST Action TU1208.

\section{References}

[1] J. D. Joannopoulos et al., Photonic Crystals: Molding the Flow of Light, 2nd ed. Princeton: Princeton University Press, 2008.

[2] F. Yang and Y. Rahmat-Samii, Electromagnetic Band Gap Structures in Antenna Engineering. New York: Cambridge University Press, 2009.

[3] M. Shahidul Alam, N. Misran, B. Yatim, and M. Tariqul Isla, "Development of electromagnetic band gap structures in the perspective of microstrip antenna design", Int. J. on Antenn. and Propag., vol. 2013, Article ID 507158, pp. 1-22, 2013.

[4] M. Thevenot et al., "Directive photonic band-gap antennas", IEEE Trans. on Microwave Theory and Techniq., vol. 47, no. 11, pp. 2115-2122, 1999.

[5] R. Gonzalo et al., "Enhanced Patch antenna performance by suppressing surface waves using photonic band-gap structures", IEEE Trans. on Microwave Theory and Techniq., vol. 47, no. 11, pp. 2131-2138, 1999.

[6] D. Sievenpiper et al., "Antennas on high-impedance ground planes", in IEEE MTT-S Int. Microwave Symp. Digest, Anaheim, CA, USA, 1999, vol. 3, pp. 1245-1248.

[7] A. R. Weily, L. Horvath, K. P. Esselle, B. C. Sanders, and T. S. Bird, "A planar resonator antenna based on a woodpile EBG material", IEEE Trans. on Antenn. and Propag., vol. 53, no. 1, pp. 216-223, 2005.

[8] R. Chantalat, L. Moustafa, M. Thevenot, T. Monediere, and B. Jecko, "Low profile EBG resonator antennas", Int. J. on Antenn. and Propag., vol. 2009, Article ID 394801, 2009.

[9] Z. Liu et al., "Research progress on Fabry-Perot resonator antenna", J. of Zhejiang Univ. Sci. A, vol. 10(8), 583-588, 2009.

[10] J. Gomez, A. Tayebi, J. R. Almagro, I. Gonzalez, and F. Catedra, "Design and optimization of an EBG antenna with an efficient electromagnetic solver", Int. J. on Antenn. and Propag., vol. 2012, Article ID 427178, 8 pages, 2012.

[11] F. Frezza, L. Pajewski, E. Piuzzi, C. Ponti, and G. Schettini, "Radiation-enhancement properties of an X-band woodpile EBG and its application to a planar antenna", Int. J. on Antenn. and Propag., vol. 2014, Article ID 729187, 15 pages, 2014.

[12] L. Pajewski, L. Rinaldi, and G. Schettini, "Enhancement of directivity using 2D-electromagnetic crystals near the band-gap edge: a full-wave approach", Progress in Electromag. Res., vol. 80, pp. 179-196, 2008. 
[13] S. Ceccuzzi, L. Pajewski, C. Ponti, and G. Schettini, "Directive EBG antennas: a comparison between two different radiating mechanisms", IEEE Trans. on Antenn. and Propag., vol. 62, no. 10, pp. 5420-5424, 2014.

[14] Y. Lee, X. Lu, Y. Hao, S. Yang, J. R. G. Evans, and C. G. Parini, "Low-Profile Directive Millimeter-Wave Antennas Using FreeFormed Three-Dimensional (3-D) Electromagnetic Bandgap Structures", IEEE Trans. on Antenn. and Propag., vol. 57, no. 10, pp. 2893-2903, 2009.

[15] H. Liu, S. Lei, X. Shi, and L. Li, "Study of antenna superstrates using metamaterials for directivity enhancement based on FabryPerot Resonant cavity", Int. J. on Antenn. and Propag., vol. 2013, Article ID 209741, 10 pages, 2013.

[16] V. Jandieri, K. Yasumoto, and Y. Liu, "Directivity of Radiation of a dipole source coupled to cylindrical electromagnetic bandgap structures", J. Opt. Soc. of America B, vol. 29, no. 9, pp. 2622-2629, 2012.

[17] K. M. Ho, C. T. Chan, C. M. Soukoulis, R. Biswas, and M. Sigalas, "Photonic band gaps in three dimensions: new layer-by-layer periodic structures", Solid State Commun., vol. 89, no. 5, pp. 413-416, 1994.

[18] H. S. Sözüer and J. P. Dowling, "Photonic band calculations for woodpile structures", J. of Modern Optics, vol. 41, no. 2, pp. 231-239, 1994.

[19] F. Frezza, L. Pajewski, E. Piuzzi, C. Ponti, and G. Schettini "Design and fabrication of a 3D-EBG superstrate for patch antennas", in Proc. 39th Eur. Microwave Conf. EuMC 2009, Rome, Italy, 2009, pp. 1496-1499.

[20] F. Frezza, L. Pajewski, and G. Schettini, "Full wave characterization of three-dimensional electromagnetic band-gap structures", IEEE Trans. on Nanotechnol., vol. 5, no. 5, pp. 545-553, 2006.

[21] F. Frezza, L. Pajewski, and G. Schettini, "Periodic defects in 2DPBG materials: full-wave analysis and design", IEEE Trans. on Nanotechnol., vol. 2, no. 3, pp. 126-134, 2003.

[22] F. Frezza, L. Pajewski, and G. Schettini, "Numerical investigation on the filtering behaviour of 2D-PBGs with multiple periodic defects", IEEE Trans. on Nanotechnol., vol. 4, no. 6, pp. 730-739, 2005.

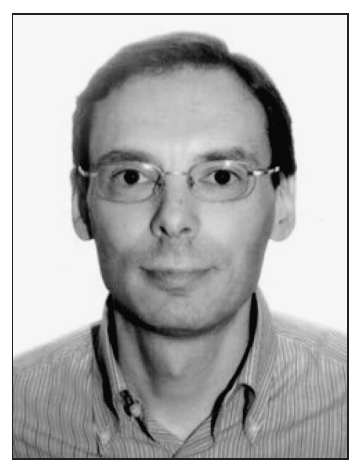

Emanuele Piuzzi received the M.Sc. (cum laude) and Ph.D. degrees in Electronics Engineering from the Sapienza University of Rome, Rome, Italy, in 1997 and 2001, respectively. He is currently an Associate Professor of Electrical and Electronic Measurements with the Department of Information Engineering, Electronics and Telecom- munications, Sapienza University of Rome. He has coauthored over 100 publications. His current research interests include the measurement of complex permittivity of materials, time domain reflectometry applications, biomedical instrumentation design, and evaluation of human exposure to electromagnetic fields. Dr. Piuzzi is a member of the Italian Group of Electrical and Electronic Measurements, and the Italian Electrotechnical Committee.

E-mail: emanuele.piuzzi@uniroma1.it

La Sapienza University of Rome

Department of Information Engineering, Electronics and Telecommunications

via Eudossiana 18

00184 Rome, Italy

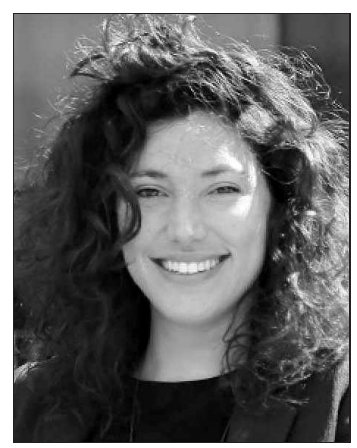

Giorgia V. Rossi received her $\mathrm{Ph} . \mathrm{D}$. in Electrical and Electronic Engineering from Imperial College London, United Kingdom, in 2017. During her Ph.D., as part of the Communications and Signal Processing group, her research focused on improving the performance of current vehicle-to-vehicle communication standards, to enable applications in road safety and hybrid networking. This research was funded by the Defence Science and Technology Laboratory (DSTL) and further involved a collaboration with Toshiba Research Europe Limited (TREL), in the form of a research placement in 2016. Previously, she received an M.Sc. in Electronic Engineering for Telecommunications from Roma Tre University, Italy. Her final M.Sc. thesis focused on the design of vehicular ad hoc networks and was carried out in cooperation with the Imperial College London. She is currently working at Cisco Systems. E-mail: giorgia.rossi12@imperial.ac.uk Imperial College London South Kensington Campus London SW7 2AZ, United Kingdom

Lara Pajewski - for biography, see this issue, p. 29.

Fabrizio Frezza - for biography, see this issue, p. 23.

Marian Marciniak - for biography, see this issue, p. 35. 